\title{
Chronic Sixth Nerve Palsy due to Compression by the Anterior Inferior Cerebellar Artery
}

\author{
Bhranavi Arulratnam, Daniel M. Mandell, Jonathan A. Micieli $\mathbb{D}^{\circ}$
}

Keywords: Sixth nerve palsy, Anterior inferior cerebellar artery, Neurovascular compression

A 65-year-old healthy woman presented with a 15-year history of binocular horizontal diplopia worse when looking left. She had previously been thoroughly investigated multiple times for a left sixth nerve palsy (6NP) 15 years ago and had three normal magnetic resonance imaging (MRI) scans of the brain/ orbits with contrast, normal acetylcholine receptor antibodies, normal thyroid function tests, normal cerebrospinal fluid, and normal nerve conduction studies and single-fibre electromyography. She was treated with prism glasses, which resulted in resolution of her symptoms in primary position.

She re-presented due to worsening of her double vision and was symptomatic with her prism glasses in primary position for 6 weeks. She had an incomitant esotropia that measured 20 prism diopters in primary position with a left abduction deficit of $50 \%$ of normal (Figure 1A). Due to the numerous previously normal investigations and chronic course, neurovascular compression was suspected. 3-Tesla MRI of the brain, including a high-resolution, three-dimensional, T2-weighted SPACE sequence (voxel size $0.5 \mathrm{~mm}$ isotropic) covering the posterior fossa, showed contact between the anterior inferior cerebellar artery (AICA) and the left abducens nerve at the nerve root entry zone (Figure 1B-E), which was the likely cause of her 6 NP. She was treated with Fresnel prisms and her symptoms resolved and the orthoptic measurements remained stable for 6 months.

Neurovascular compression is a well-recognized but rare cause of $6 \mathrm{NP} .{ }^{1}$ This may occur from aneurysms, ${ }^{2}$ dolichoectactic $^{3}$ or relatively normal-appearing vessels ${ }^{1}$ in an aberrant location. This patient had a relative large AICA in contact with the left abducens nerve at the root entry zone, and the mechanism of the 6NP was likely related to mass effect and pulsatility on the abducens nerve in this location. There was also contact of the right AICA with the right abducens nerve, but the right AICA was a much smaller artery than the left AICA, and had no effect on the function of this nerve. The normal anatomic location of the AICA has been shown to be away from the root entry zone of the abducens nerve. ${ }^{4}$ It is recognized that pathology occurs only when vessels are in contact with a cranial nerve at or proximal to the root entry zone, which is the transition zone between the central and peripheral myelin and an area that lacks perineurium and epineurium and is particularly vulnerable to continued pulsatile pressure. ${ }^{5,6}$

Five previous cases of neurovascular compression of the AICA on the abducens nerve resulting in a $6 \mathrm{NP}$ have been described. ${ }^{6-10}$ The location of the compression was at the root entry zone in four cases, and the abducens nerve was sandwiched between a persistent trigeminal artery and the AICA in one case. ${ }^{9}$ Every patient experienced spontaneous improvement, and four out of five cases had recurrent sixth nerve palsies and the number of episodes varied from 4 to $11 .^{6-9}$ The time to recovery varied from 2 to over 4 months. ${ }^{7,10}$ No neurosurgical treatment was pursued in any of the cases. This case demonstrates that neurovascular compression of the abducens nerve from the AICA can also have a chronic course.

\section{ACKNowledgments}

None.

\section{Disclosures}

The authors have no conflicts of interest to disclose.

\section{Statement of Authorship}

Conception and design (JAM), data acquisition (BA, DMM, JAM), manuscript draft and preparation (BA, DMM), critical analysis (JAM, DMM), final approval (BA, DMM, JAM).

From the Kensington Vision and Research Centre, Toronto, Ontario, Canada (BA, JAM); Department of Medical Imaging, Toronto Western Hospital, Toronto, Ontario, Canada (DMM); Department of Ophthalmology and Vision Sciences, University of Toronto, Toronto, Ontario, Canada (JAM); Division of Neurology, Department of Medicine, University of Toronto, Toronto, Ontario, Canada (JAM)

Received August 12, 2019. Date of Acceptance September 28, 2019. Correspondence to: Dr. Jonathan A. Micieli, Kensington Vision and Research Centre, 340 College Street, Suite 501, Toronto, Ontario M5T 3A9, Canada. Email: jmicieli@kensingtonhealth.org 

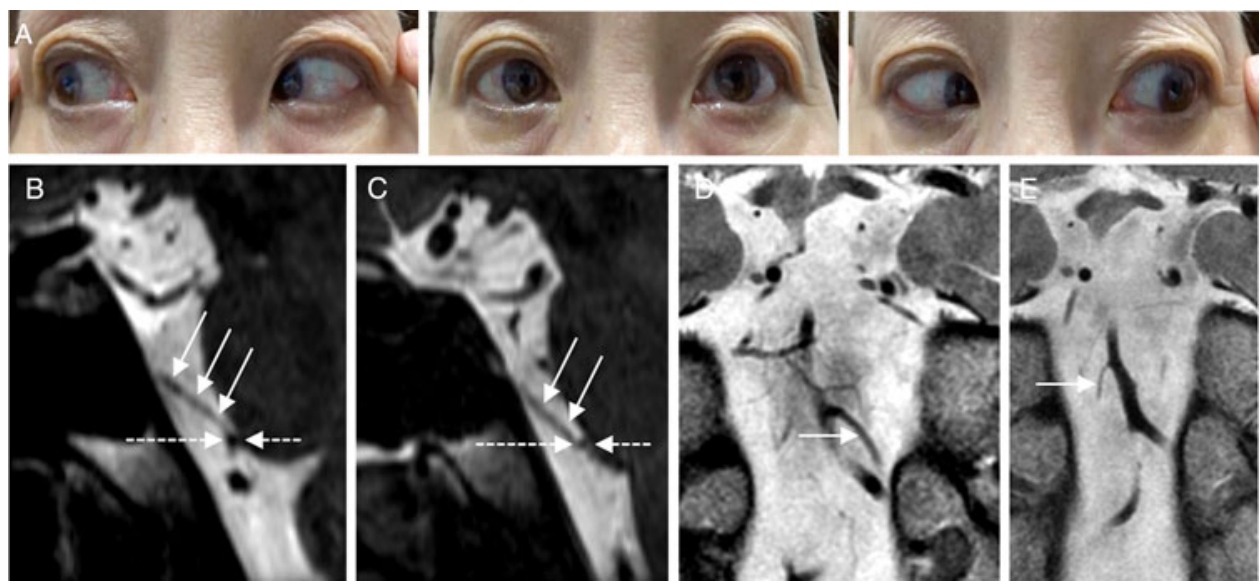

Figure 1: External photo demonstrating a limitation of abduction in the left eye (A). Sagittal-oblique reformat $(B)$ of an axial three-dimensional T2-weighted sequence shows the left abducens nerve cisternal segment (solid arrows) in contact with the left anterior inferior cerebellar artery (AICA) (dotted arrow) at the nerve root entry zone. Sagittal-oblique reformat $(C)$ of the same pulse sequence $(C)$ shows the right abducens nerve (solid arrows) in contact with a much smaller right AICA (dotted arrow). Coronal T2weighted sequence shows that the left AICA (D, arrow) is much larger than the right AICA (E, arrow).

\section{REFERENCES}

1. Tsai TH, Demer JL. Non-aneurysmal cranial nerve compression as cause of neuropathic strabismus: evidence from high-resolution magnetic resonance imaging. Am J Ophthalmol. 2001;152: 1067-73.

2. Er U, Fraser K, Lanzino G. Isolated unilateral sixth nerve palsy as a presenting symptom of cerebral aneurysms. Report of two cases. Neuroradiol J. 2007;20:81-4.

3. Zhu Y, Thulborn K, Curnyn K, Goodwin J. Sixth cranial nerve palsy caused by compression from a dolichoectatic vertebral artery. J Neuroophthalmol. 2005;25:134-5.

4. Delion M, Dinomais M, Mercier P. Arteries and veins of the cerebellum. Cerebellum 2017;16:880-912.

5. Janetta PJ. Observations on the aetiology of trigeminal neurolagia, hemifacial spasm, acoustic nerve dysfunction and flossopharyngeal neuralgia. Neurochirugica 1977;20:145-54.
6. Sandvand KA, Ringstad G, Kerty E. Periodic abducens nerve palsy in adults caused by neurovascular compression. J Neurol Neurosurg Psychiatry. 2008;79:100-2.

7. Kato H, Nakajima M, Ohnaka $Y$, Ishihara K, Kawamura M. Recurrent abducens nerve palsy associated with neurovascular compression. J Neurol Sci. 2010;295:135-6.

8. Linn J, Schwarz F, Reinisch V, Straube A. Ophthalmoplegic migraine with paresis of the sixth nerve: a neurovascular compression syndrome? Cephalagia. 2008;28:667-70.

9. Nakamagoe K, Mamada N, Shiigai M, Shimizu K, Koganezawa T, Tamaoka A. Recurrent isolated abducens nerve paresis associated with persistent trigeminal artery variant. Intern Med. 2012; 51:2213-6.

10. Taniguchi A, Ii Y, Kobayashi H, Maeda M, Tomimoto H. Bilateral abducens nerve palsy by compression from bilateral anterior inferior cerebellar artery. J Neurol. 2011;258:2271-3. 